\title{
VINCULACIÓN YTRANSFERENCIA TECNOLÓGICA EN LA UNIVERSIDAD AUTÓNOMA DE ENTRE RÍOS
}

Mgter. Lic. Norberto Muzzachiodi (*)

\section{Resumen}

A fines del año 2012 acontece un hito trascendental en la vida de la UADER, su normalización, que da impulso a diversas acciones estratégicas hacia el interior de la misma fortaleciéndola institucionalmente y académicamente, y hacia el exterior robusteciendo los lazos institucionales con el conjunto de actores de la sociedad entrerriana. Para dar cumplimiento a las políticas de vinculación y transferencia, se crea la Dirección de Vinculación y Transferencia Tecnológica, dependiente de la Secretaría de Extensión Universitaria, que absorve la UVT UADER. En el Proyecto Institucional de la Universidad aprobado por Resolución No 086-13 del Consejo Superior se definen las lineas básicas de la gestión y transferencia en el ámbito de la Universidad. Se destaca en la actualidad el seguimiento y administración de proyectos de distintas convocatorias y fuentes de financiamiento dentro de los que se destacan 14 financiados por el MINCTe IP; 17 por el ME SPU; 3 con Fondos Provinciales ademas de los Servicios a terceros. La Universidad participa de la Red VITEC que articula las áreas de vinculación tecnológica de las universidades nacionales e institutos universitarios que integran el Consejo Interuniversitario Nacional (CIN) y es Socio Fundador de la
RED ALCUE junto a 36 Universidades Iberoamericanas, que promueve un observatorio de noticias para difundir las actividades en relación con la Unión Europea que efectúan las Universidades. Se participa activamente de dos proyectos internacionales como lo son la Red Universitaria para el Fomento de Empresas Sociales y Tecnológicas -RUFESyT- y la Red de Incubadoras del Mercosur -RIUM-. Por ultimo la UADER firmo un Convenio con Consejo de Investigaciones Científicas y Técnicas mediante la cual se aprobó por Res. 3776/13 a la UVT UADER a realizar actividades por cuenta y orden del CONICET en el marco de la Ley 23.877 de Fomento de Innovación Tecnológica.

Palabras Claves: UVT - Vinculación Transferencia - Desarrollo Regional.

\section{Introducción}

Se definió a la Unidad de Vinculación, en la Ley Nacional $N^{\circ} 23.877 / 90$ como un "ente no estatal constituido para la identificación, selección y formulación de proyectos de investigación y desarrollo, transmisión de tecnología y asistencia técnica. Representa el núcleo fundamental del sistema, aportando su estructura jurídica para facilitar la gestión, organización y gerenciamiento de los proyectos" (Infoleg, 2014).

(*) Dirección de Vinculación y Transferencia Tecnológica. Unidad de Vinculación. Tecnológica. Universidad Autónoma de Entre Ríos. Entre Ríos, Argentina. Av. Ramírez 1143. 3100. Paraná. Entre Ríos - nmuzzachiodi@uader.edu.ar 0343-154749925 
La Unidad de Vinculación Tecnológica de la Universidad Autónoma de Entre Ríos (UVT - UADER) cuenta con habilitación nacional, otorgada por la Agencia Nacional de Promoción Científica y Tecnológica mediante Resolución $\mathrm{N}^{\circ} 193$, del 13 de agosto de 2008, dependiendo funcionalmente de la Dirección de Vinculación y Transferencia Tecnológica que según estructura orgánica de la Universidad depende de la Secretaria de Extensión Universitaria.

\section{Historia joven de una Universidad Provincial}

La Universidad Autónoma de Entre Ríos (UADER) fue creada por Ley $\mathrm{N}^{\circ}$ 9.250 de la Provincia de Entre Ríos, sancionada el 8 de junio de 2000, siendo una de sus misiones fundamentales la creación $\mathrm{y}$ transferencia del conocimiento, que se ha ido adecuando a la complejidad y demandas de la sociedad actual.

De este modo ofrece una política de apertura y vinculación de la sociedad, poniendo a disposición de los distintos sectores de la sociedad entrerriana con una marcada presencia territorial 85 carreras que se dictan en 15 ciudades de la provincia, con un total de 100 títulos que se otorgan, entre licenciaturas, profesorados, tecnicaturas y traductorados, a través de sus cuatro facultades: Humanidades, Artes y Ciencias Sociales; Ciencia y Tecnología; Ciencias de la Gestión; y Ciencias de la Vida y de la Salud, caracterizada por su carácter público, gratuito, inclusivo y con compromiso social.

\section{Vinculación y Transferencia Tecnoló- gica en la UADER.}

La UVT - UADER marcó una nueva impronta de trabajo para la UADER, en cuanto a la gestión y administración de proyectos específicamente relacionados con los sectores productivos y sociales de la Provincia.

A fines del año 2012 acontece un hito trascendental en la vida de la UADER, su normalización. Este hecho dio impulso a diversas acciones estratégicas hacia el interior de la misma fortaleciéndola institucionalmente y académicamente, y hacia el exterior robusteciendo los lazos institucionales con el conjunto de actores de la sociedad entrerriana.

Para dar cumplimiento a las nuevas demandas de vinculación con el medio, a principios del año 2013, el Rector Bioing. Aníbal Javier Sattler crea la Dirección de Vinculación y Transferencia Tecnológica (DVyTT - UADER), dependiente de la Secretaría de Extensión Universitaria, de la cual paso a depender la UVT - UADER.

\section{Unidad de Vinculación Tecnológica - UADER}

La UVT - UADER acompaña a los potenciales beneficiarios de recursos económicos en la formulación, presentación y administración de proyectos de diversas fuentes de financiamiento, con temáticas que atienden diversos aspectos, intereses y demandas de la sociedad, abarcando temáticas que van desde discapacidad, emprendedorismo, salud, ambiente, desarrollo de las capacidades locales (Muzzachiodi et al. 2014; Tornero et al. 2014; Canavelli et al. 2014).

Se destaca en la actualidad el seguimiento y administración de proyectos de distintas convocatorias y fuentes de financiamiento dentro de los que se pueden clasificar a grandes rasgos en:

A. Fondos nacionales del Ministerio de Ciencia, Tecnología e Innovación Productiva: 1 MINCyT PFIF 2005, 1 MIN- 
CyT PFIF 2006, 1 PICT CCLIP 2008; 1 MINCyT ASETUR 2009; 3 MINCyT DETEM 2009; 1 PROCODAS 2013; 2 MINCyT DETEM 2011; 1 MINCyT PFIF 2009; 1 MINCyT PFIP ESPRO 2010; 2 PROCODAS 2013.

B. Fondos nacionales del Ministerio de Educación de la Nación: 4 Manuel Belgrano SPU 2013; 3 Ingeniero Enrique Mosconi SPU 2013; 3 de Fortalecimiento UVT SPU; 1 Fortalecimiento de Redes Interuniversitarias V; 2 SPU Universidad, Diseño y Desarrollo; 1 Programa Universidad y Transporte 2014.

C. Fondos Provinciales: 5 Vice gobernación Provincia de Entre Ríos 2010; 2 Programa de I+D+i: Desarrollo de un Cluster en Biociencias aplicadas a la Salud, el Ambiente y la Agroindustria 2010.

D. Servicios a terceros: 1 FUCOFA FCyT; 1 UCAR - FCyT.

La Universidad participa de la Red VITEC que articula las áreas de vinculación tecnológica de las universidades que integran el Consejo Interuniversitario Nacional (CIN) que promueve la inserción de las universidades en el sector productivo.

Integra el Foro de la Región Centro, que es uno de los cuatro foros que conforman el Consejo de la Sociedad Civil de la Región Centro de Argentina, integrado por instituciones y entidades no gubernamentales de las provincias de Córdoba, Entre Ríos y Santa Fe, junto a 17 Universidades Públicas y Privadas de las mencionadas provincias.

Por otro lado, la UVT - UADER dirige y participa en proyectos de redes interuniversitarias nacionales e internacionales en donde el emprendedorismo e incubación de empresas son ejemplos de ello, como lo son la Red Universitaria para el
Fomento de Empresas Sociales y Tecnológicas -RUFESyT- y la Red de Incubadoras del Mercosur -RIUM-.

Se destaca la UADER por ser Socia Fundador de la RED ALCUE junto a 36 Universidades Iberoamericanas, que promueve un observatorio de noticias para difundir las actividades en relación con la Unión Europea que efectúan las Universidades.

Por ultimo la UADER firmo un Convenio con Consejo de Investigaciones Científicas y Técnicas mediante la cual se aprobó por Res. 3776/13 a la UVT UADER a realizar actividades por cuenta y orden del CONICET en el marco de la Ley 23.877.

\section{Proyección territorial}

La continuidad de las políticas nacionales y provinciales de Ciencia, Tecnología e Innovación, permiten impulsar una economía basada en el conocimiento y en este propósito, nuestra UVT cumplirá un rol significativo dentro de la Provincia de Entre Ríos y Región Centro.

Se reconoce la "tercera misión" basada en la extensión universitaria y compromiso con la comunidad (D'Este et al. 2009) en el marco de una universidad social, contrapuesta al modelo impuesto en los 90 (Puigross, 2006), no aceptando una Universidad que se limite al circuito de la teoría y práctica de las formas de producción que buscan reducir la vida a las leyes de oferta y demanda capitalista (Malagón Plata, 2004) regidas por la idea de mercado con principios mercantilistas e utilitarios deshumanizantes (Ansaldi, 1995), y por el contrario, impulsamos acciones tendientes a convalidar la concepción de la ciencia y la tecnología como expresiones de un proceso complejo del ser humano, en cuanto a la significa- 
ción y transformación que producen en su entorno; representando, por tanto, un acto sociocultural (Lara, 2009).

E1 Rector de la UADER aseveró que "las universidades somos parte de la dinámica social y nos estamos pensando cada vez más en nuestro vínculo con las necesidades históricas en términos de conocimiento, pero también de construcción política y de acompañamiento a los procesos de gestión pública” (Sattler, 2013).

En la última década las universidades forman parte de un país sustentable económica y socialmente, que puso a la ciencia y a la tecnología en el centro del modelo de desarrollo nacional (Recalde, 2013), pero debemos evitar la transferencia tecnológica ciega mediante el cual el sistema científico argentino desarrolla conocimientos con potencial aplicación industrial que es apropiado exógenamente por empresas extranjeras (Codner et al. 2012).

E1 Rector Sattler ha manifestado analizando el primer año de gestión de normalización que la Universidad Autónoma de Entre Ríos es una institución educativa peculiar, sostenida por un entramado sociocultural diverso que ha sabido transformar fuertes dificultades, por estar habitado por numerosas expresiones y contar con un desarrollo territorial ciertamente amplio, en un capital que le ha posibilitado, en muy corto tiempo, generar una alianza potente con la sociedad y sus estrategias de desarrollo.

\section{BIBLIOGRAFÍA}

Ansaldi, W. 1995. Gobernabilidad democrática y desigualdad social. Estudios Sociales. 9: 7-24.

Canavelli, A. Muzzachiodi, N. y J. Tornero. 2014. La Universidad y su compromiso con la inclusión social. 10 p. En: Ponencias III Jornadas de Extensión MERCOSUR 2014. Eje: Políticas Públicas para la promoción y la inclusión social. Tandil, 10 y 11 de abril de 2014.

Tornero, J.; Muzzachiodi, N. y A. Canavelli 2014. Promoción de la cultura emprendedora e innovadora de base social y tecnológica - UADER. 14 p. En: Ponencias III Jornadas de Extensión MERCOSUR 2014. UNICEN. Tandil, 10 y 11 de abril de 2014

Codner, D.G.; Becerra, P. y A. Díaz. 2012. Blind Technology Transfer or Technological Knowledge Leakage: a Case Study from the South. Journal of Technology Management \& Innovation 7(2): 184-195

D’Este, P.; Castro Martínez, E. y J. Molas-Gallart. 2009. Documento de base para un "Manual de Indicadores de Vinculación de la universidad con el entorno socioeconómico". INGENIO (CSIC-UPV). Valencia, $26 \mathrm{p}$.

INFOLEG. Ley N² 23.877. Promoción y fomento de la innovación tecnológica. Centro de Documentación e Información, Ministerio de Economía y Finanzas Públicas.

Lara, L. M. 2009. Acerca de la potencialidad secuestrada de la Ciencia y Tecnología. Por una idea del desarrollo multidimensional. Pp 17 - 22 En: Figueroa Delgado, S. A.; Sánchez Daza, G. A. Vidales Carmona. 2009. La ciencia y tecnología en el desarrollo. Una visión desde América latina. Universidad Autónoma de Zacatecas. México, 86 p. Malagón Plata, L. A. 2004. Universidad y sociedad: pertinen- 
cia y educación superior. Cooperativa Editorial Magisterio. Bogotá, 211 p.

Muzzachiodi, N.; Tornero; J.; Canavelli, A.y G. Guzmán Ruiz. 2014. La Universidad y su compromiso con el Medio Ambiente. 12 p. En: Ponencias III Jornadas de Extensión MERCOSUR 2014. Tandil, 10 y 11 de abril de 2014.

Puiggrós, A. 2006. Qué pasó en la educación Argentina: breve historia desde la conquista hasta el presente. Galerna. Buenos Aires, 224 p.

Recalde, A. 2013. Universidad y Desarrollo Popular. pp 55-59. En: La Universidad en 30 años de democracia. Ministerio de Educación. Buenos Aires, 43 p.

Sattler, A. J. 2013. Creando puentes. pp 35-39. En: La Universidad en 30 años de democracia. Ministerio de Educación. Presidencia de la Nación. Buenos Aires, 43 p. 